\title{
Brightness Observations of the Sun
}

\author{
By JEFFREY R. KUHN
}

\author{
National Solar Observatory/Sunspot, NM 88349, USA
}

There are few solar surface brightness observations with sufficient accuracy to have direct bearing on the question of the origin of the Sun's bolometric luminosity and irradiance changes (the correlative observations have been reviewed elsewhere in these proceedings). This paper contrasts these observations with a model for the solar variability and illustrates our clear need for high precision surface photometry, in combination with helioseismic data and numerical experiments.

\section{Introduction}

It is important to distinguish between irradiance and luminosity. I use the term "irradiance" to refer to the brightness, in a given direction (in the plane of the ecliptic) from the Sun, or in a specific direction from a particular region of the photosphere. The "luminosity" is then the integrated flux over all outward directions and over some area. Thus, the net energy budget of a part of the photosphere or the entire Sun is then reflected in our understanding of the solar luminosity - not the irradiance. All measurements to date, whether spatially resolved or integrated full-disk observations, only directly yield the irradiance, and such changes, alone, do not imply luminosity changes. From the astrophysical perspective the reason to study these "total irradiance" variations is to learn about the mechanism that causes them. I believe we won't achieve this until we know more about how the solar cycle works, and it may be that we learn quite a bit about it from the irradiance measurements. In any case it is clear that a "good" physical model of the irradiance data must also account for the other solar cycle changes. We can expect the helioseismic cycle variations to be a very useful constraint in this regard. A previous review by Kuhn (1992) describes one perspective on this problem. This discussion elaborates on some of the ideas from that paper.

The short term (day-to-day) and long term (yearly) total irradiance variations have magnitudes of a few times $10^{-4}$ of the mean solar irradiance. Spatially resolved observations must have comparable accuracy to see these changes. Since the ground-based experiments must peer through an atmosphere with an overall extinction variation that is typically two orders of magnitude larger - we do well to give up on the task of seeing the total solar irradiance variability (at this level) from the ground. Yet, the ground-based data may very well tell us how the irradiance changes come about, since it is possible to obtain this level of photometric precision differentially. Then, from additional assumptions (e.g. that the surface brightness in quiet regions of the photosphere doesn't change), we can "bootstrap" an argument for spatially integrating the differential photometry data to obtain the total irradiance. Comparison with ERB or ACRIM satellite experiments, which do have good absolute precision, then allow a test of the assumptions that go into interpreting the differential photometry. Note that progress here depends on interpreting high precision differential measurements in combination with high precision absolute solar photometry.

We took this approach (Kuhn et al. 1988) to compare limb temperature observations to ACRIM data. With the assumption that the effective color temperature of the photosphere outside of the active latitude bands and the solar equator doesn't change during the cycle, it could be shown that the solar luminosity was changing over long (yearly) timescales. This limb photometry was limited, in that the spatial resolution was 
low and only the outer 20 arcseconds of the limb could be observed using the "oblateness" telescope. Also, the latitudinal photometric variations that were detected could not be uniquely identified, or easily associated with other solar phenomena (like the photospheric network, or "polar" faculae). On the other hand recent independent measurements (Kroll 1994) have described indirect evidence for effective limb-temperature variations comparable to these.

On shorter timescales the first non-correlative direct observations of irradiance changes were obtained by Nishikawa \& Hirayama (1990) using a CCD camera system. Although they needed to fit and subtract largescale spatial trends from their full-disk photometry, they did achieve high photometric precision at the pixel scale. With the assumption that the quiet photosphere was not variable they were able to show that most of the short term solar irradiance changes are associated with sunspots and active regions.

Recently Lin (1993) and Lin \& Kuhn (1992) used visible and IR sensitive array detectors to try to identify both the large and small scale contributions to the total irradiance signal. Lin also found a latitudinal variation similar to the Kuhn et al. (1988) results, but lacked sufficient spatial resolution to identify the latitudinal bolometric changes with, for example, a photospheric network irradiance signal. Topka (1993) has used very high spatial resolution observations, in relatively small fields-of-view, to argue that the network bright points do not account for the observed long term irradiance changes. His conclusions depend on the assumption that the local mean photosphere doesn't vary in brightness between regions where he measures the network contrast. It is notable that there is a large body of high precision, but small field-of-view, differential photometric data aimed at determining sunspot contrast (e.g. Fowler \& Foukal 1983 for measuring CZ diffusivity) or facular contrast (e.g. Lawrence 1988 for pinning down facular models) but these observations do not directly yield constraints on the irradiance mechanism.

\section{Local observations, global conclusion}

The solar limb photometry implies that on a long timescale the solar luminosity changes. Although these photometric results need to be confirmed, the data imply that the Sun is about $0.1 \%$ brighter near solar maximum than near minimum. On shorter timescales we know that irradiance changes tend to be anticorrelated with surface magnetic fields. We cannot say on these timescales if there is a correlation between such magnetic fields and luminosity. The problem of course, is that the total luminosity associated with photospheric features is difficult both to measure and to model. The radiant energy budget of a flux tube obviously depends on the timescale over which you study it, while the direct measurement of the total emergent flux depends on simultaneous observations from many directions, or on waiting for it to rotate around the Sun (during which time it may evolve). Local observations of feature contrast (cf Topka 1993) versus angle can, in principle, be integrated to obtain the luminosity change due to the feature, but the calculation is flawed if the surrounding photosphere (which the contrast is measured with respect to) changes brightness.

Without high precision full-disk differential photometry, one method for measuring the magnetic feature luminosity is to use the ACRIM timeseries data, which is an integral of the full-disk brightness. This approach was used (Kuhn 1992) to show that there is a general anticorrelation between the average photospheric magnetic field and the irradiance on short timescales. Presumably this is just the effect seen in individual timeseries records where irradiance drops as sunspots rotate across the disk (cf. Willson \& Hudson, 1988). There is a strong statistical signal in the ACRIM timeseries that shows an irradiance excess one quarter solar rotation before and after the irradiance 


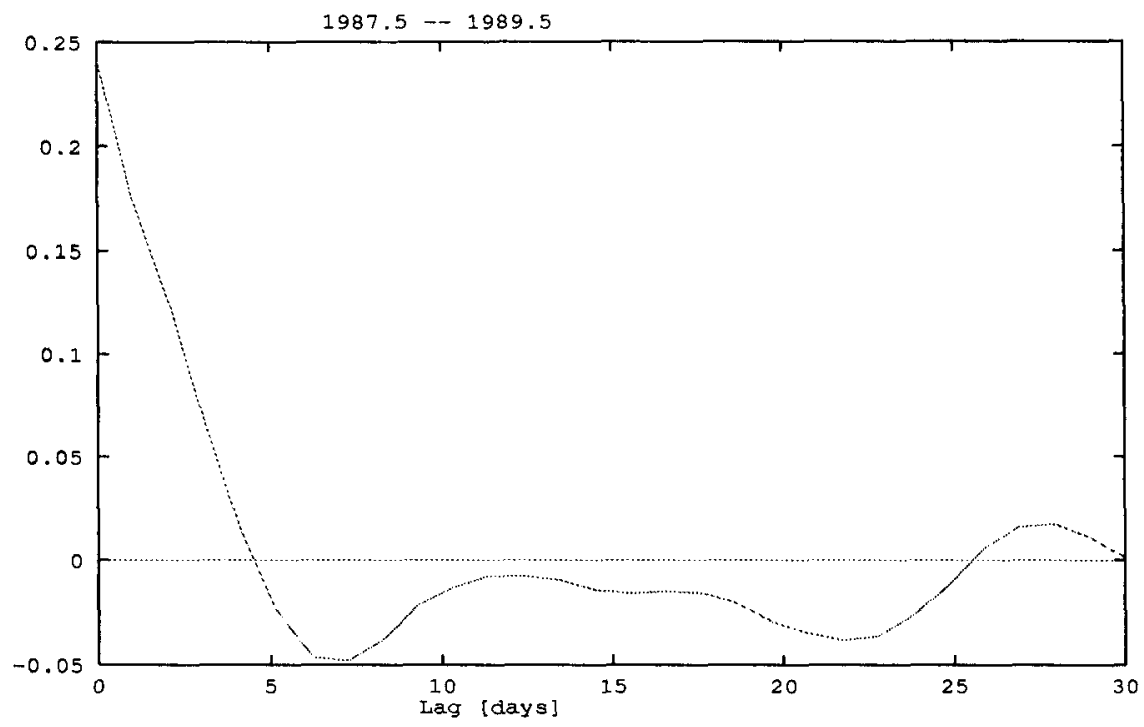

FIGURE 1. ACRIM autocorrelation.

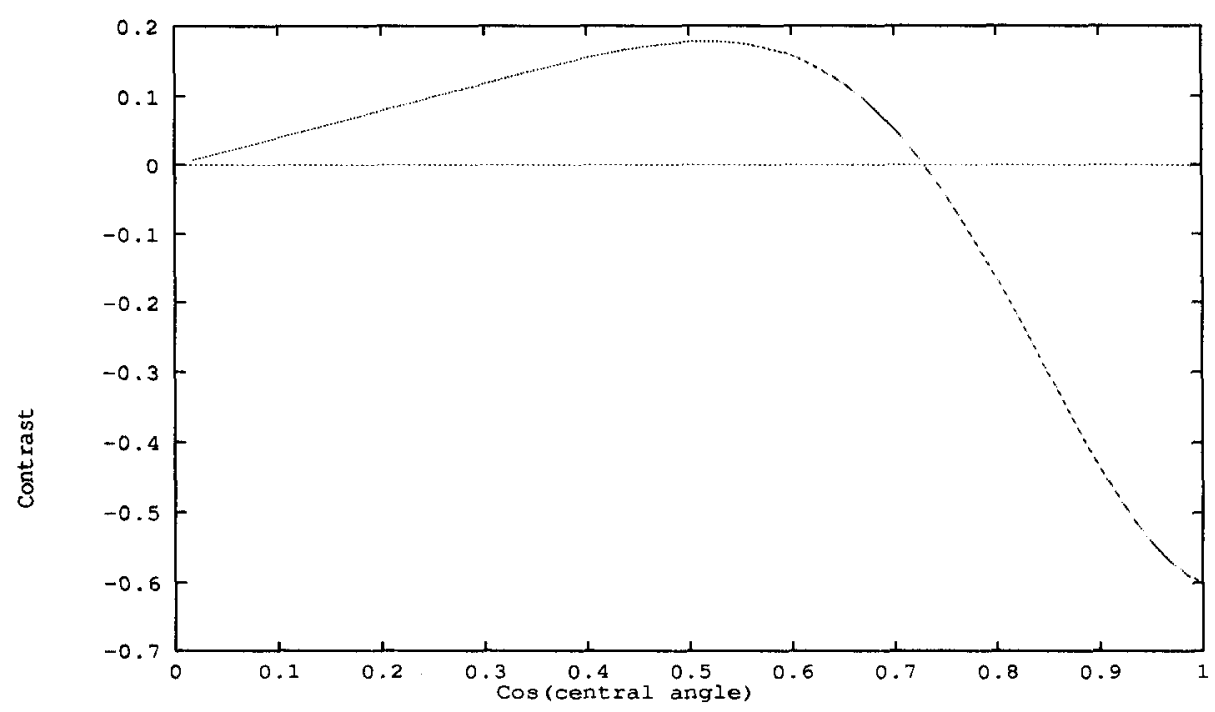

Figure 2. Active Region Contrast Function.

dips. This suggests that the short-term anticorrelated irradiance signal may not imply a corresponding decreased magnetic feature luminosity. The flux lost from the normal direction in active region magnetic features is redistributed at large angles from the local normal. This conclusion seems to describe the collective effect of all magnetic elements, without distingushing faculae and sunspots. Thus, individual spots and faculae may have very different radiation patterns, but the collective effect of active regions shows a clear flux redistribution signal in the ACRIM autocorrelation analysis.

Figure 1 shows the autocorrelation of ACRIM irradiance data after solar minimum until the end of the timeseries record in 1990. A 60 day moving mean was subtracted 


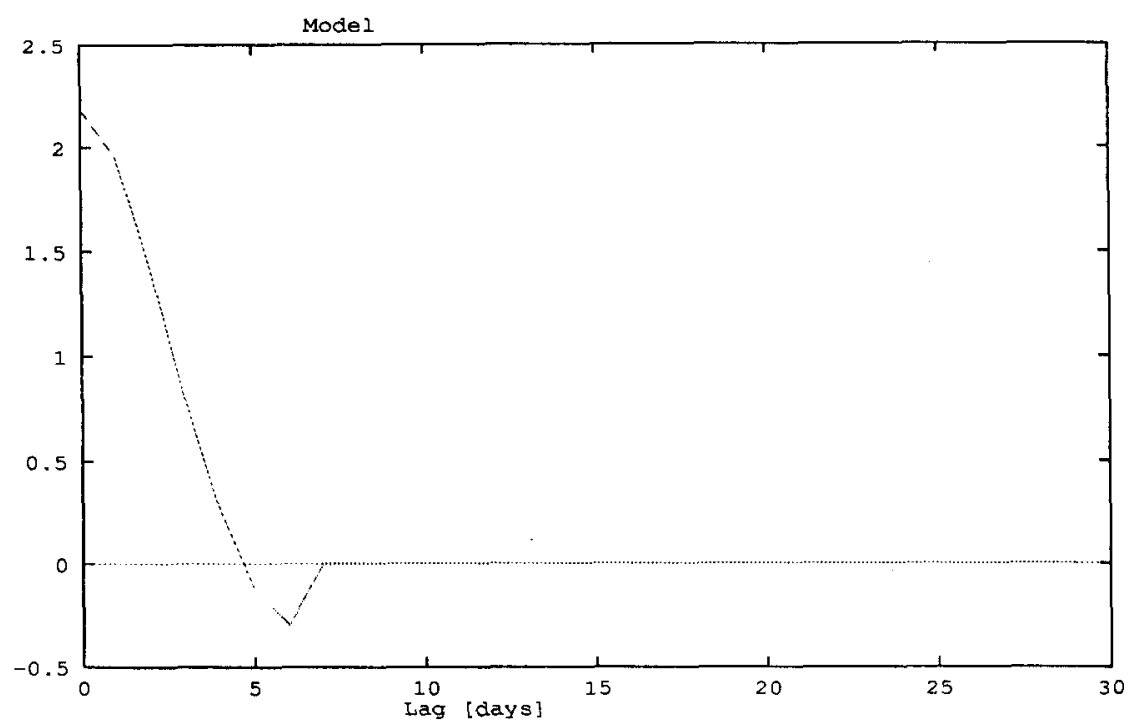

FIGURE 3. Model autocorrelation.

to remove the slow solar cycle trend. The graph shows the negative peak near a lag of $1 / 4$ of a rotation (6-7 days). This data is probably too noisy to uniquely invert the autocorrelation function for the effective "active region contrast function" but Figure 2 illustrates a simple "forward" calculation where I hypothesised a 2 parameter contrast function and varied the parameters until I reproduced (by "eye") the autocorrelation displayed in Figure 1. The particular contrast function plotted in Figure 2 has the form $d I / I=\left\{c-\exp \left[-\left(\frac{(1-\mu)^{2}}{2 \mu_{0}^{2}}\right)\right]\right\} \times \mu$ and for parameters $c=0.4$ and $\mu_{0}=0.3$ the angular integral of this function implies essentially no change in total luminosity, although this contrast function does exhibit an irradiance decrease near disk center $(\mu=1)$ and falls to zero at the extreme limb. It implies the autocorrelation shown in Figure 3, which is quite similar to the actual data plotted in Figure 1. The conclusion from this example is that the filtered ACRIM timeseries, even though it shows short-term irradiance drops, is consistent with an "active region" contrast function that implies no short-term solar luminosity changes. Testing this conclusion requires precise full-disk photometry over a long enough (several rotation periods) time series. A statistical measure of active region luminosity changes could be extracted from the rotationally modulated surface brightness records. To compare one timeseries record with the next, it is critical that the local active region brightness be known with respect to the solar surface brightness far from the region, i.e. global (full-disk) photometry is needed.

\section{What causes long-term luminosity changes?}

Considering the large fractional amplitude of the magnetic changes during the cycle it is likely that the luminosity variations are directly related to the magnetic field evolution. Does the luminosity vary as an incidental consequence of fields poking through the photosphere, which allow radiation to escape more easily through the lower gas density and opacity in flux tubes, or does the luminosity change reflect variations in entropy deeper in the convection zone (CZ), or below?

The rather small solar cycle change in the acoustic mode frequencies which are sensi- 


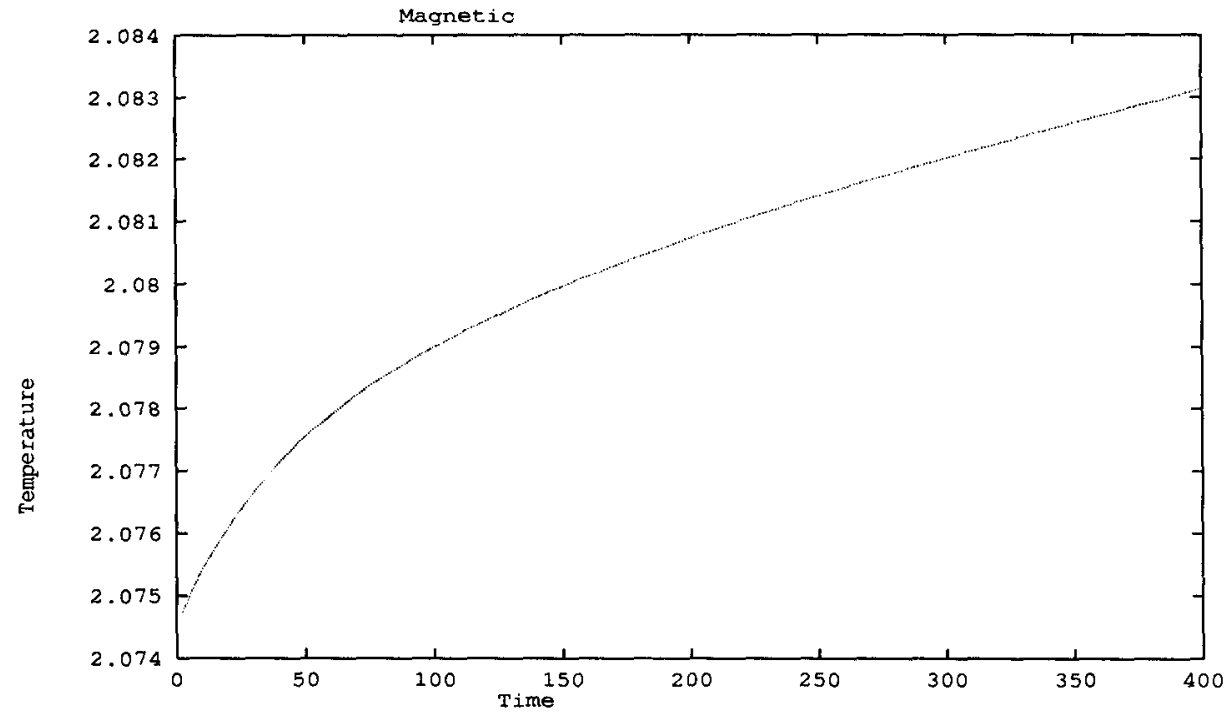

Figure 4. Temperature $\left(10^{6} \mathrm{~K}\right)$ vs. time (yrs).

tive to the solar core conditions rules out fundamental changes in the radiative interior temperature structure as the source of the yearly luminosity variations we see at the photosphere. The amplitude of the temperature variations in the energy generating core would need to be enormous (fractional changes of order unity) to appear as 11 year variations at the surface, since the radiative diffusion time exceeds $10^{5}$ years.

In the simplest model photospheric magnetic fields may act as "shorts" to radiative diffusion. The magnetic field locally decreases gas density and increases the photon free path. In this simple picture most of the excess energy radiated through the "antiplug" is compensated by a deficit surrounding the short. Since the solar atmosphere is exponentially stratified most of the energy transported out the antiplug comes from horizontal diffusion. Thus in an exponentially stratified diffusive model the total outgoing energy flux is only marginally enhanced. In other words the radiation pattern of the region changes, but its total luminosity change is small (Kuhn 1992).

Of course the solar convection in and around faculae and sunspots makes a detailed solution a much more difficult problem. For example, if the effect of convective energy transport were to isotropically couple much of the convection zone (for example, a large "effective" conductivity) then the "antiplugs" described above could change the energy content of the convection zone while changing the solar luminosity (Spruit 1994). In conflict with this assumption, we find from numerical simulations of energy transport in a realistic outer convection zone that convective energy transport is very anisotropic, with entropy transport vertically being at least an order of magnitude larger than horizontally. This conclusion is quite consistent with the strong velocity anisotropy observed in the numerical simulations. Precise observations of the horizontal thermal structure around magnetic regions will tell us what the entropy transport properties of the upper convection zone are really like.

If the short term irradiance changes are due to flux redistribution from photospheric magnetic fields, and they do not produce significant luminosity changes, then what causes the solar cycle luminosity variation? A deeper mechanism is suggested by the longer timescale of these changes. Whether or not an $\alpha-\omega$ dynamo is at work to produce 
a)

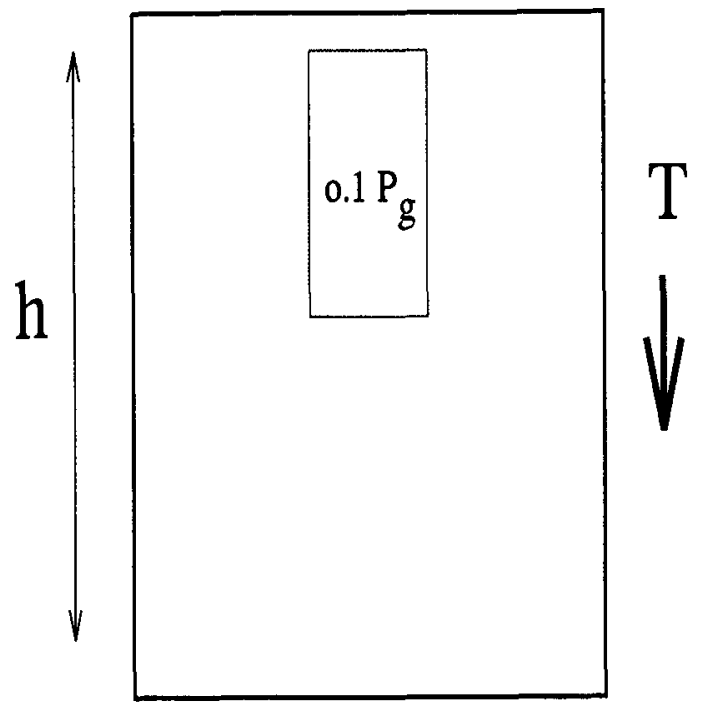

b)

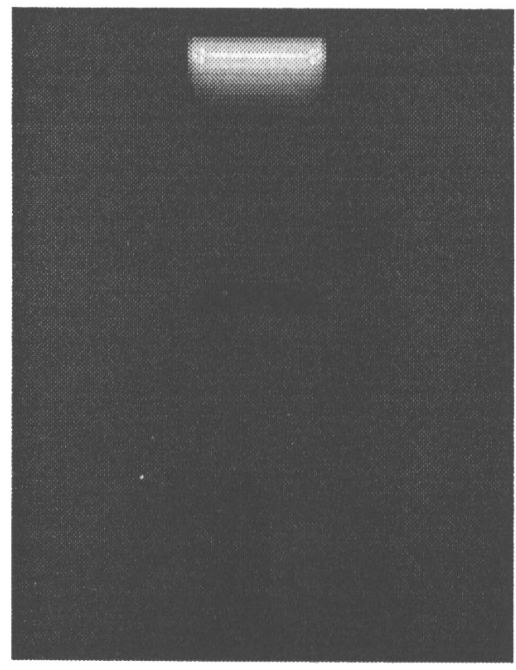

c)

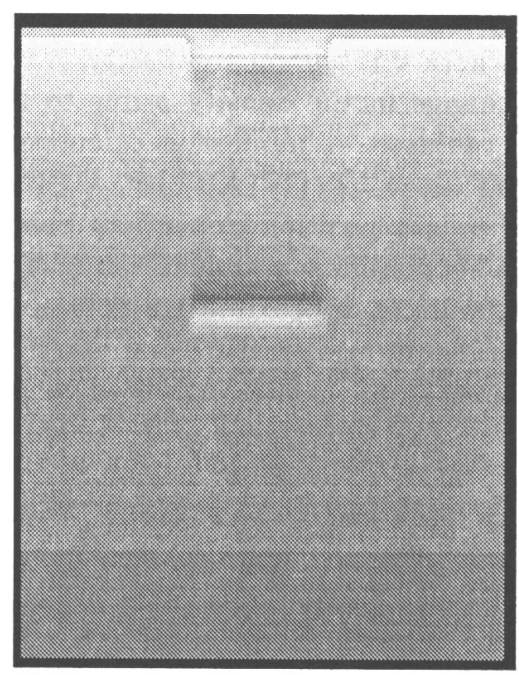

Figure 5. a) Simulation geometry, b) Fractional temperature change after 11 years, c) Temperature gradient after 11 years.

the magnetic cycle, it is likely that the surface magnetic flux originates from near the base of the convection zone. Since helioseismic observations of the interior differential rotation place the largest radial shear just below the $\mathrm{CZ}$ it seems that the toroidal field is amplified there. I argue below that magnetised fluid that originates in the radiative 
zone should have a higher entropy than surrounding layers. This excess entropy could emerge on a longer timescale than the magnetic flux and be responsible for the increase in the solar luminosity observed near the peak of the activity cycle.

A thin sheath of magnetic flux immediately below the base of the $\mathrm{CZ}$ must grow hotter than corresponding fluid with the same horizontal stratification. Radiation below the flux sheet diffuses through the magnetised region with a longer photon free-path because the local density is decreased by the local magnetic pressure. Thus the top of the magnetised fluid is hotter than the surroundings. Consequently the magnitude of the temperature gradient also increases above the top surface of the flux sheet. If the flux sheet is immediately below (and parallel to) the base of the convection zone the effect of the increased temperature gradient is to locally lower the bottom of the convection zone to the surface of the flux sheet. Convective overshoot or magnetic bouyancy can then operate to bring magnetic flux, which originated below the $\mathrm{CZ}$ boundary, into the $\mathrm{CZ}$ and finally into the photosphere. This radiative instability not only causes the magnetised fluid to have a higher entropy than unmagnetised fluid, but it also provides a mechanism for bringing flux from the $\mathrm{RZ}$ into the $\mathrm{CZ}$. In effect the bottom surface of the $\mathrm{CZ}$ must be "corrugated" latitudinally by the magnetic perturbation. This effect can be looked for in helioseismic data, which already give very accurate measurements of the mean depth of the CZ (Christensen-Dalsgaard et al. 1991).

The timescale for heating the magnetised fluid depends on the vertical extent of the sheet and the field strength. The growth time $\tau$ is approximately

$$
\tau=\frac{3 \pi \kappa \lambda \rho}{16 \sigma T \Delta|d T / d z|}
$$

where $\kappa$ is the mean opacity, $\lambda$ is the vertical scale of the flux sheet, $\Delta$ is the fractional change in the density $\rho$ due to the magnetic field, and $d T / d z$ is the local temperature gradient. A numerical calculation illustrates how this effect may operate. We solve $d T / d t=k T / P \nabla\left(K T^{4} / P \quad \nabla T\right)$ subject to the ideal gas law with $P=P_{g a s}+P_{B}$ when $P_{B}$ is a position dependent constant corresponding to the "local magnetic pressure". This nonlinear problem was first solved with $P_{B}$ everywhere zero and constants $k$ and $K$ chosen to approximate a volume near the top of the RZ in the Sun. A $64 \times 64$ grid with constant temperature boundary conditions on the top and bottom surfaces and periodic horizontal surfaces was used with a standard relaxation technique. Into this equilibrium solution a magnetic field was added (corresponding to $10 \%$ of the gas pressure) near the surface of the volume. Figure 5a shows the relative size of the pressure scale height, $h$, and the magnetised volume. Figure 4 shows how the temperature at the top surface of the volume changes. Over 11 years the temperature increases by about 1000 degrees, or about $0.05 \%$. Figure $5 \mathrm{~b}$ shows a greyscale image of the fractional temperature change in the volume. The top surface gets warmer while the lower magnetic boundary is cooler. Figure $5 \mathrm{c}$ shows the vertical temperature gradient, also plotted as a greyscale image. The gradient increases toward the top boundary where the fluid becomes convectively unstable. Immediately above the magnetised volume the gradient also increases, tending to lower the base of the $\mathrm{CZ}$ above magnetic latitudes.

In this picture, photospheric magnetic flux causes the irradiance to drop as a magnetic region rotates toward disk center. This irradiance change is accompanied by an isotropic luminosity increase which is related to the total magnetic flux in the region. The excess entropy carried by magnetic flux tubes appears at the photosphere over a longer timescale than the active region growth time since the entropy excess is carried throughout the flux tube volume. How this energy diffuses to the photosphere is yet undetermined. This excess appears as a luminosity increase over longer timescales on top of the short term 
irradiance decrease. From the ecliptic plane we see the short term irradiance decrease with magnetic field while the overall magnetic activity defines an increasing irradiance "baseline" which is actually the solar-cycle luminosity change.

\section{Future observations}

Ground-based observations to come will measure small surface brightness variations over solar-cycle timescales. Interesting observations should achieve spatial resolution and photometric precision that is limited only by the atmosphere. We should expect such a dataset to help answer questions like "how do magnetic fields affect heat flow through the convection zone?" or "can we learn about how the solar cycle works from the luminosity of photospheric features?" Is the "valve" that modulates the total solar luminosity operating at the photosphere incidently as a consequence of the surface magnetic variation, or is the luminosity controlled from the base of the CZ? Answers will follow from sensitive measurements of, for example, the radiation temperature excess and its time dependence from active regions, or the latitudinal variation of the network luminosity. Such data will provide another "boundary condition" on the interpretation of helioseismic data that probe the solar interior, and another constraint on the interpretation of numerical simulations.

\section{REFERENCES}

Christensen-Dalsgaard, J., Gough, D.O. \& Thompson, M.J. 1991 The depth of the convective zone. Ap. J. 378, 413-437.

Fowler, L. \& Foukal, P. 1983 Sunspot bright rings and the thermal diffusivity of solar convection. Solar Phys. 84, 33-44.

Kroll, R. 1993 Latitudinal variation of the solar limb-darkening function. Solar Phys., in press.

Kuhn, J.R. 1992 What causes cycle related global solar changes? GONG 1992: Seismic Investigations of the Sun and Stars (PASP: San Francisco), 27-40.

Kuhn, J.R., Libbrecht, K. \& Dicke, R.H. 1988 The surface temperature of the Sun and changes in the solar constant. Science 242, 908-911.

Lawrence, J.K. 1988 Multicolor photometric observations of facular contrast. Salar Phys. 116, 17-32.

Lin, H.: 1993 Precision IR and visible photometry II. Ap. J, submitted.

Lin, H. \& Kuhn, J.R. 1992 Precision IR and Visible solar photometry. Solar Phys. 141, 1-26.

Nishikawa, N. \& Hirayama, T. 1990 Facular structures derived from precise two-color contrast observations. Solar Phys. 74, 315.

Spruit, H. 1994 Theoretical interpretation of solar and stellar irradiance variations. In The Sun as a Variable Star: Solar and Stellar Irradiance Variations (ed. J.M. Pap, C. Fröhlich, H.S. Hudson \& S.K. Solanki). Cambridge University Press, in press.

Topka, K. 1993 High resolution solar photometry. Solar Physics Division of AAS, in press.

Willson, R.C. \& Hudson, H.S.: 1988 Solar luminosity variation in cycle 21 . Nature 332, 810-812. 